\title{
UNIQUE FACTORIZATION OF IDEALS INTO NONFACTORABLE IDEALS
}

\author{
H. S. BUTTS
}

The purpose of this note is to prove a theorem which shows a connection between the definition of a prime ideal in classical algebraic number theory and the usual definition of a prime ideal. A proper ideal in an integral domain with unit element is an ideal different from the unit ideal and the zero ideal. An ideal $A$ will be called nonfactorable provided $A$ is a proper ideal and $A=B C$ (where $B$ and $C$ are ideals) implies that either $B$ or $C$ is the unit ideal.

THEOREM. If $J$ is an integral domain with unit such that every proper ideal of $J$ is either a nonfactorable ideal or can be factored uniquely into a product of nonfactorable ideals, then $J$ is a Dedekind domain and the nonfactorable ideals are prime in the usual sense.

Proof. Let $P$ be a proper prime ideal of $J$ and $p \neq 0$ be an element of $P$. There exist nonfactorable ideals $N_{1}, \cdots, N_{n}$ in $J$ such that $(p)=N_{1} \cdot \cdots \cdot N_{n}$. Let $N_{i}$ be an arbitrary member of the collection $N_{1}, \cdots, N_{n}$. Since $(p)$ is an invertible ideal, it follows that $N_{i}$ is an invertible ideal and consequently $N_{i}$ is finitely generated (see $[1$, p. 272]).

Let $x$ be any element of $J$ such that $x$ is not an element of $N_{i}$. Since $N_{i}$ is finitely generated, then $N_{i}+(x)$ is finitely generated. The cancellation law for ideals is valid in $J$ (an obvious consequence of the unique factorization property) and therefore finitely generated ideals are invertible (see [2, p. 13]). Hence $N_{i}+(x)$ is invertible and since $N_{i}+(x) \supset N_{i}$ there exists an ideal $Q$ in $J$ such that $\left[N_{i}+(x)\right] \cdot Q$ $=N_{i}$. It is clear that $N_{i}+(x)=J$ and $N_{i}$ is a maximal ideal.

Since $P$ is a prime ideal and $P \supset N_{1} \cdot \cdots \cdot N_{n}$ it follows that $P \supset N_{i}$ for some $i$ and therefore $P$ is invertible. Hence every proper prime ideal of $J$ is invertible and $J$ is a Dedekind domain (see [3, Theorem 7, p. 33]).

\section{REFERENCES}

1. Oscar Zariski and Pierre Samuel, Commutative algebra, Vol. 1, Van Nostrand, Princeton, N. J., 1958.

2. Heinz Prüfer, Untersuchungen über Teilbarkeitseigenshaften in Korpern, J. Reine Angew. Math. 168 (1932), 1-36.

3. I. S. Cohen, Commutative rings with restricted minimum condition, Duke Math. J. 17 (1950), 27-42.

\section{Louisiana State University}

Received by the editors July 8, 1962 and, in revised form, October 9, 1962. 